\title{
Untersuchungen über das Nervensystem von Hexaurus merkli J. Frivaldzsky (Coleoptera, Bathysciinae)
}

\author{
MICHAIL KWARTIRNIKOV*
}

Dieser Artikel ist die Fortsetzung einer Untersuchungsserie über das Nervensystem und das endokrine System der bulgarischen troglobionten Käfer. Es wird damit versucht ein wenig Licht auf das interessante Problem zu werfen, welche Veränderungen das Übergehen zum Höhlenleben mit sich gebracht hat.

Es ist bereits von vielen Troglobionten bekannt, daß die Lobi optici, die Nervi optici völlig zurückgebildet sind. Bei den meisten troglobionten Käfern wird auch von einer erheblichen Konzentration des zentralen Nervensystems berichtet. Diese Erscheinungen gilt es auch hier zu suchen. Zusammen mit dem Nervensystem ist es aber interessant das endokrine System zu untersuchen, da beide voneinander schwer zu trennen sind.

\section{A T E R I A L U N D M E T H O D E N}

Das untersuchte Material stammt aus einer Höhle nahe der Bergspitze Schipka im Zentralbalkan. Es wurde im ganzen Jahr außer im Winter gesammelt, damit ein vollständigeres Bild über die endokrine Tätigkeit entsteht, falls irgend eine jahreszeitlich bedingte Dynamik existiert. Die Tiere wurden sofort mit dem Fang in der Bouinschen Flüssigkeit fixiert. Die Einbettung erfolgte mittels gewöhnlicher Parafintechnik in Hartparafin. Die Tiere wurden total geschnitten bei einer Schnittdicke von 6 Mikron für die gewöhnlichen und 20 Mikron für die Restaurationsschnitte. Da die untersuchte Art sehr klein ist (ca. $3 \mathrm{~mm}$ Länge) habe ich es bevorzugt das Nervensystem nach histologischen Schnitten zu rekonstruieren, statt freizupräparieren. Die Restaurationsschnitte wurden mit Hämatoxylin nach Ehrlich-Eosin gefärbt. Die Darstellung der neurosekretorischen Zellen geschah durch die Paraldehyd-Fuxin-Färbung. Da die wichtigsten Strukturen des Oberschlundganglions bereits nach der Paraldehyd-Fuxin-Färbung gut sichtbar waren, verzichtete ich auf spezifische Methoden für Darstellung von Nervenstrukturen.

Die Ausrechnung des prozentuellen Anteils der Corpora pedunculata und des Lobus olfactorius im Gesammtvolumen des Oberschlundganglions sowie des prozentuellen Anteils des Unterschlundganglions im Gesammtvolumen der Kopfganglien ist auf Grund einer lückenlosen Schnittserie geschehen. Sie wurde fotografiert, vergrößert und die Grenzen der genannten Strukturen wurden mit Tusche markiert. Durch Abwiegen und Herausschneiden konnten die entsprechenden Anteile in \% errechnet werden. Es ist dabei von zwei Voraussetzungen ausgegangen. Man kann mit befriedigender Genauigkeit die Konturen markieren und die Schwankungen in

\footnotetext{
* Zoologisches Institut, I Boul. Russki, Sofia, Bulgarien.
} 
der Dicke des fotografischen Papiers sind unbedeutend. Die Schrumpfungen der Gewebe infolge Fixation und Färbung sind ebenfalls zu vernachlässigen, da es sich um prozentuellen Anteil handelt und nicht um absolute Werte.
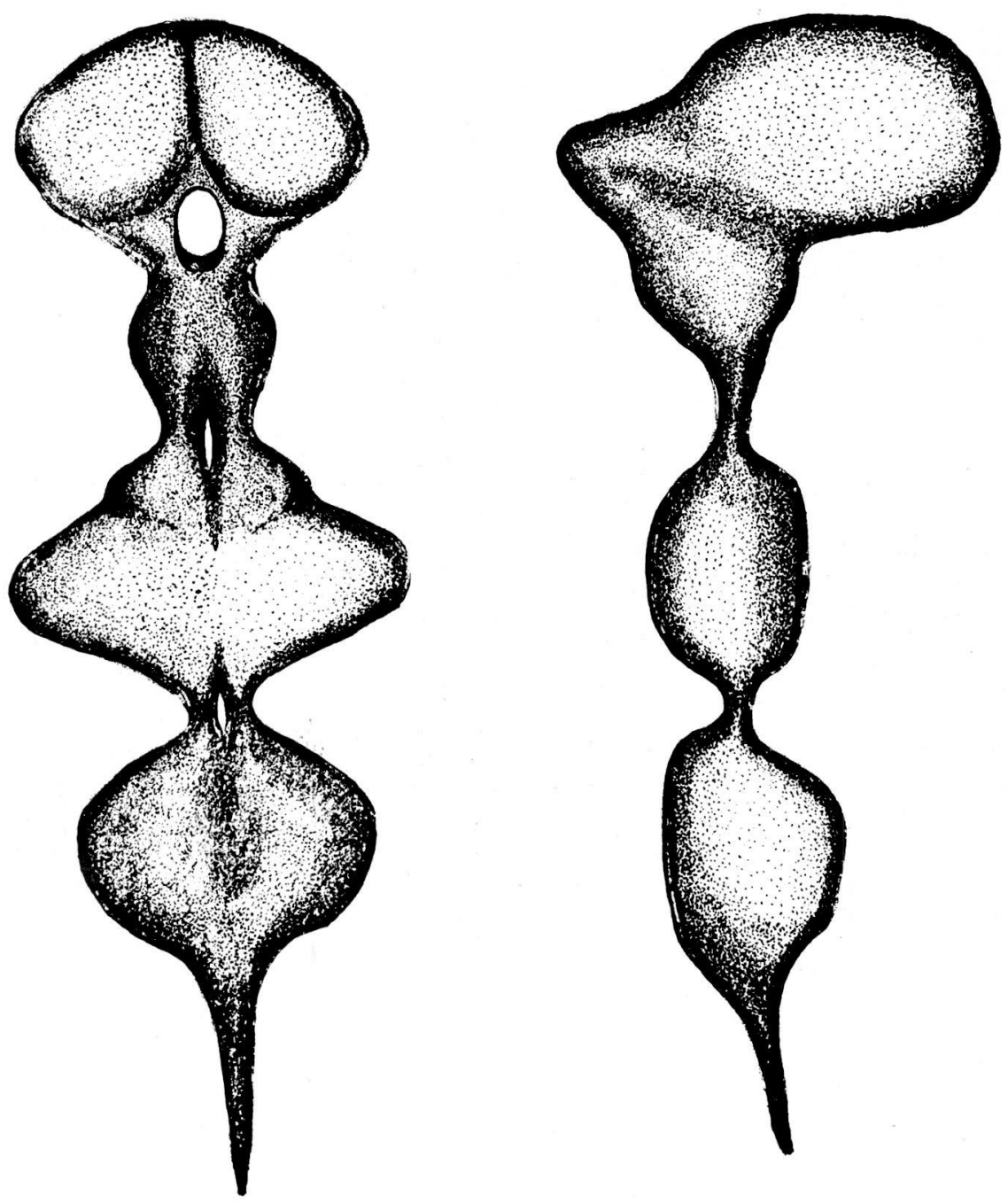

Abb. 1. Allgemeine Ansicht des zentralen Nervensystems von Hexaurus merkli. Seitlich und dorsal. Die ausgehenden Nerven nicht eingezeichnet. 


\section{R E S U L T A T E}

Das zentrale Nervensystem von Hexaurus merkli hat die Formel 2+2 / 0 (Abb. 1). Wie aus der Abbildung ersichtlich ist, besteht das cerebrale Nervensystem aus dem Ober- und Unterschlundganglion, die durch zwei flache kräftige Konnektive verbunden werden. Das Oberschlundganglion entbehrt völlig die Lobi optici und hat eine relativ einfache Form. Durch das Fehlen dieser Strukturen wird das Unterschlundganglion relativ groß und macht etwa 29,5\% von der gesammten Masse der Kopfganglien. Die histologischen Schnitte zeigten, daß sich im Unterschlundganglion zwei globuläre Gebilde befinden, die den olfactorischen Glomeruli sehr ähnlich sehen. Sie sind durch Nervenbahnen mit den Wurzelgebieten der Mandibelnerven verbunden.

Das erste Thoraxganglion erscheint als ein Synganglion. Dieser Eindruck wird auch vom histologischen Bild der Neuropilmasse gestärkt. Mit dem Unterschlundganglion und mit dem zweiten Thoraxganglion verbindet sich der erste durch doppelte Konnektive.

Das zweite Thoraxganglion liegt an der Grenze zwischen Thorax und Abdomen. Die Formel des Nervensystems wird dennoch als 2+2/0 definiert. Dies geschieht aus Gründen, die in der Diskussion erörtert werden.

Die topografische Anatomie des Oberschlundganglions zeigte interessante Tatsachen. Zuerst muss das Fehlen der Sehkomissur erwähnt werden. Ferner ist auch die Anwesenheit von Corpora pedunculata von Interesse, und zwar in einem solchen Umfang, der bei Höhlenkäfern kaum zu vermuten ist. Abbildung 2 zeigt einen seitlichen Dorsoventralschnitt auf dem Niveau der Corpora pedunculata. Ihre Form und Verlauf, verfolgt an Schnittserien, sind dem Normalfall (Weber, 1954) sehr nahe. Auf Abb. 2 ist auch eine Nervenbahn zu sehen, die von den Pilzkörpern zu den Wurzeln der Antennennerven zieht. Die assoziativen Zellen der Pilzkörper heben sich kaum von den übrigen Neuronen ab.

Der errechnete prozentuelle Anteil der Pilzkörper in der Gesammtmasse des Oberschlundganglions beträgt $5,5 \%$.

Voluminöser als die Corpora pedunculata erwiesen sich die olfactorischen Loben, die etwa 6,8\% vom Gesammtvolumen des Oberschlundganglions ausmachen.

Die Untersuchung des endokrinen Systems zeigte folgende Resultate. Es seien zuerst die Drüsen des retrocerebralen Komplexes geschildert.

Die Corpora cardiaca sind wie gewöhnlich paarige Gebilde, die beiderseits des Ösophagus liegen. Sie zeigten schwache Färbung mit Paraldehyd-Fuxin. Eng an sie angeschmiegt liegen die Corpora allata, ebenfalls paarig angelegt. Die gegenseitige Lage der Drüsen, des Gehirns und des Ösophagus zeigt Abbildung 3.

Die neurosekretorischen Zellen zeigten folgende Verteilung im zentralen Nervensystem. Im Pars intercerebralis befinden sich maximal 40 neurosekretorisch tätige Zellen. Gewöhnlich schwankt ihre Zahl aber um 20-22. Seltener sind sie weniger oder mehr. Die Zellen sind langgezogen mit deutlichem Axon. Sie färben sich nach der angegebenen Methode relativ schwach, wobei die Färbung diffus erscheint. Abbildung 4 zeigt eine Gruppe von neurosekretorischen Zellen des Pars intercerebralis. 


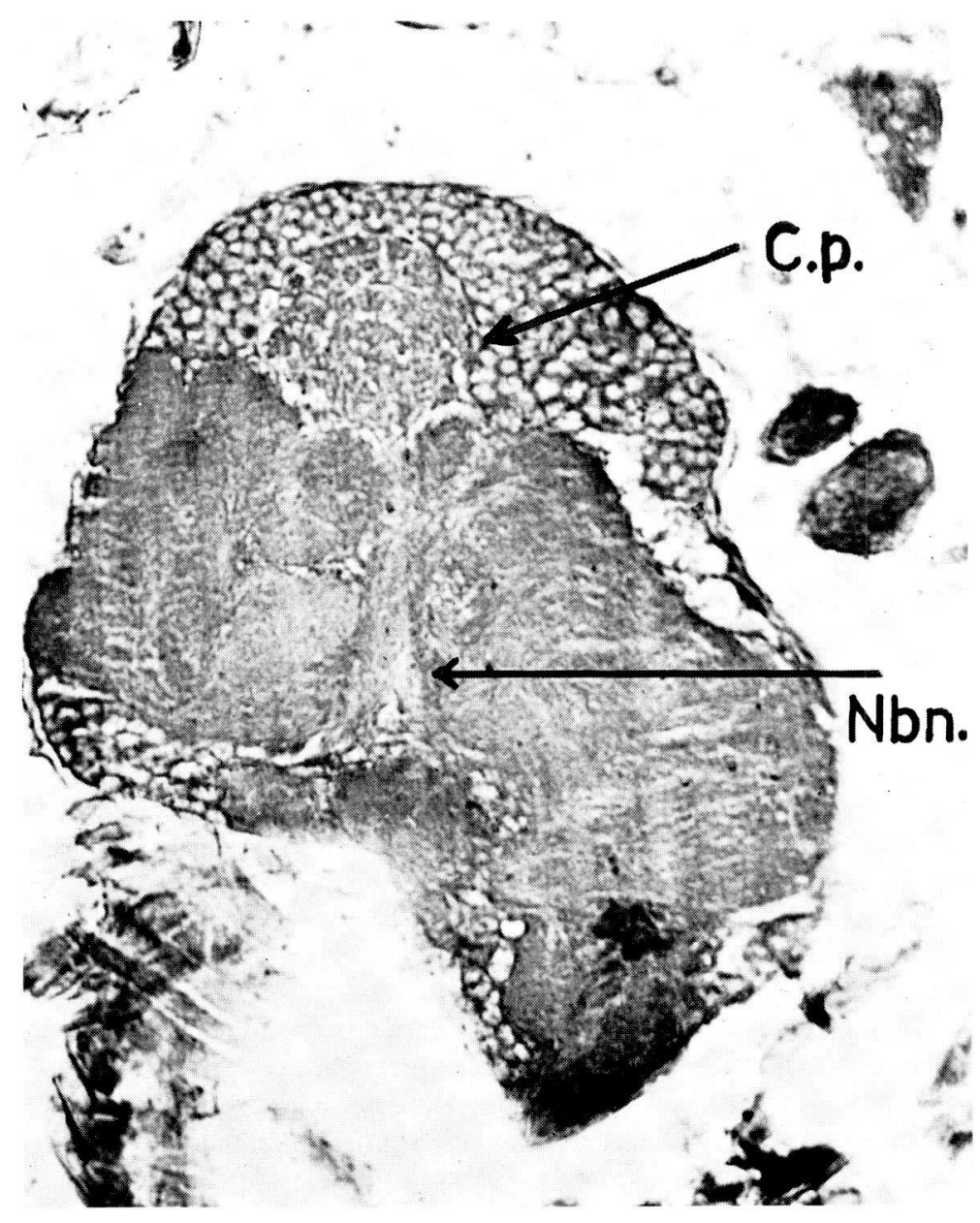

Abb. 2. Ganglion supraoesophageum seitlich geschnitten. C.p. - Corpora pedunculata. Nbn.-Nervenbahn zum Wurzelgebiet der Antennen. 


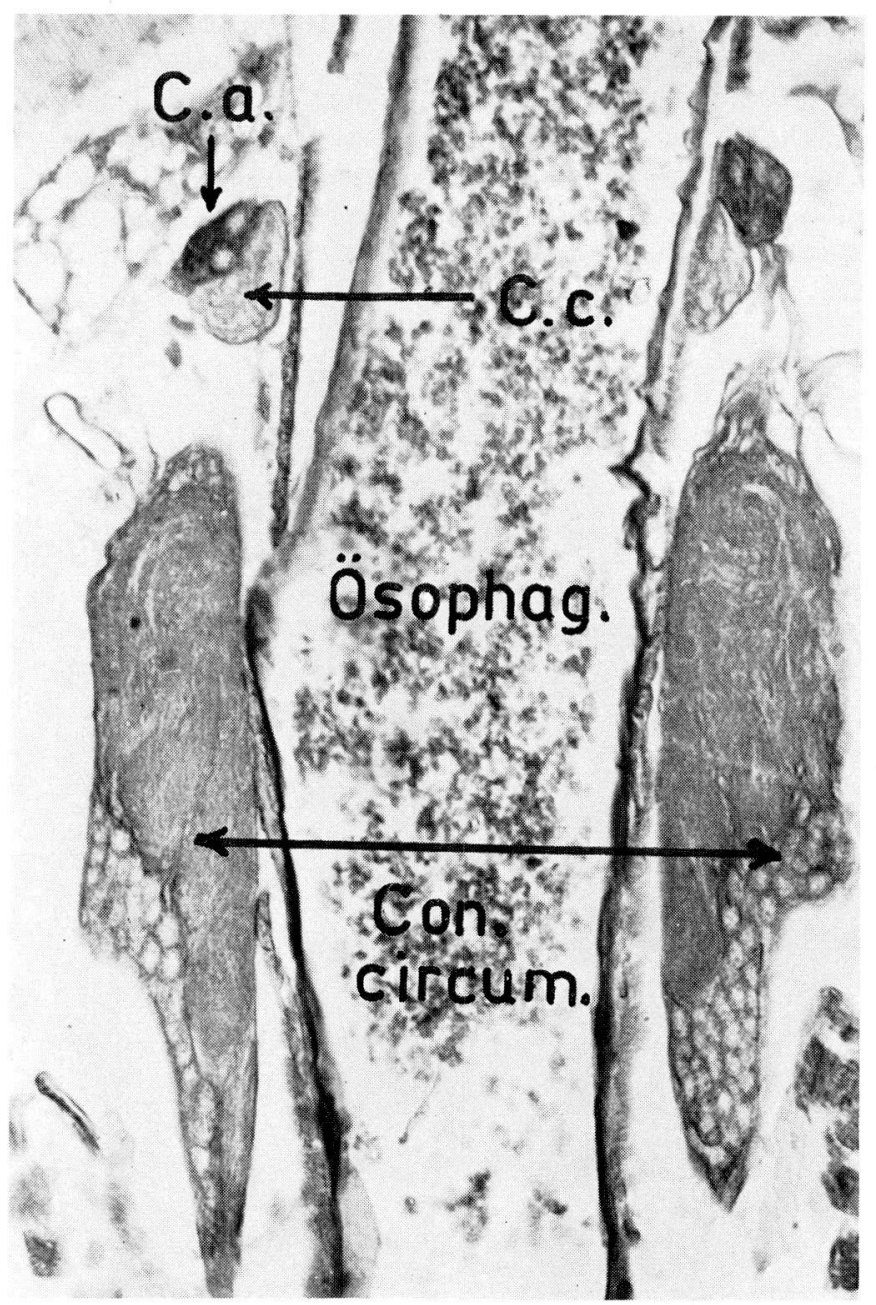

Abb. 3. Längsschnitt durch das Ganglion supraoesophageum auf dem Niveau der Connectiva circumoesophagialis - con. circum. Ösophag. - Ösophagus. C. a. - Corpora allata, C.c. - Corpora cardiaca. 
Die neurosekretorischen Zellen aus dem Unterschlundganglion zeigten ein anderes Bild. Sie sind weniger an der Zahl, sehr oft nur zwei und liegen stets dicht nebeneinander. Im Gegensatz zu den obengeschilderten Zellen färben sie sich äußerst intensiv. Die Form ist polygonal und sie liegen in der ersten Zellschicht ventral dem Unterschlundganglion an. Das Neurosekret bildet große Schollen und füllt den ganzen Zelleib aus (Abb. 5).

Außer in den Kopfganglien sind in keinem anderen Ganglion dieser Art neurosekretorische Zellen gefunden worden. Dasgleiche gilt für neurosekretorische Bahnen, die außerdem selten zu beobachten sind. Nur einmal im Oberschlundganglion von den Zellen im Pars intercerebralis ventralwärts ziehend und einmal im Unterschlundganglion in Richtung Thoraxganglien ziehende neurosekretorische Wege konnten festgestellt werden.

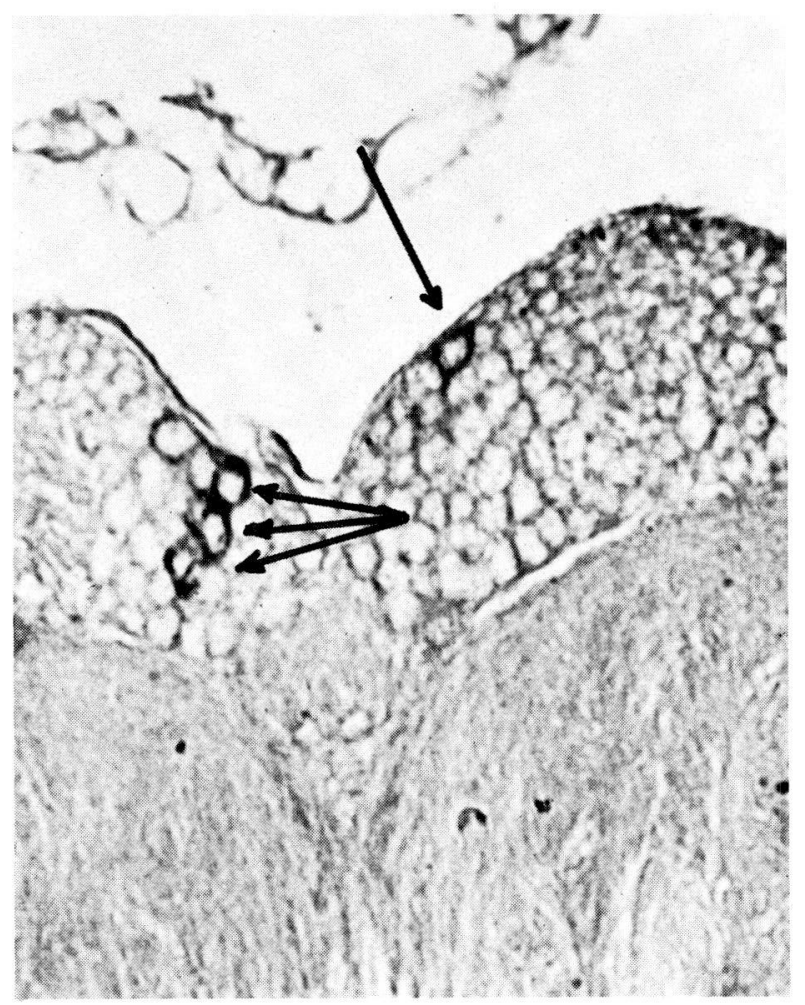

Abb. 4. Schnitt durch die Pars intercerebralis. Die Neurosekretorischen Zellen mit Pfeilen bezeichnet. 


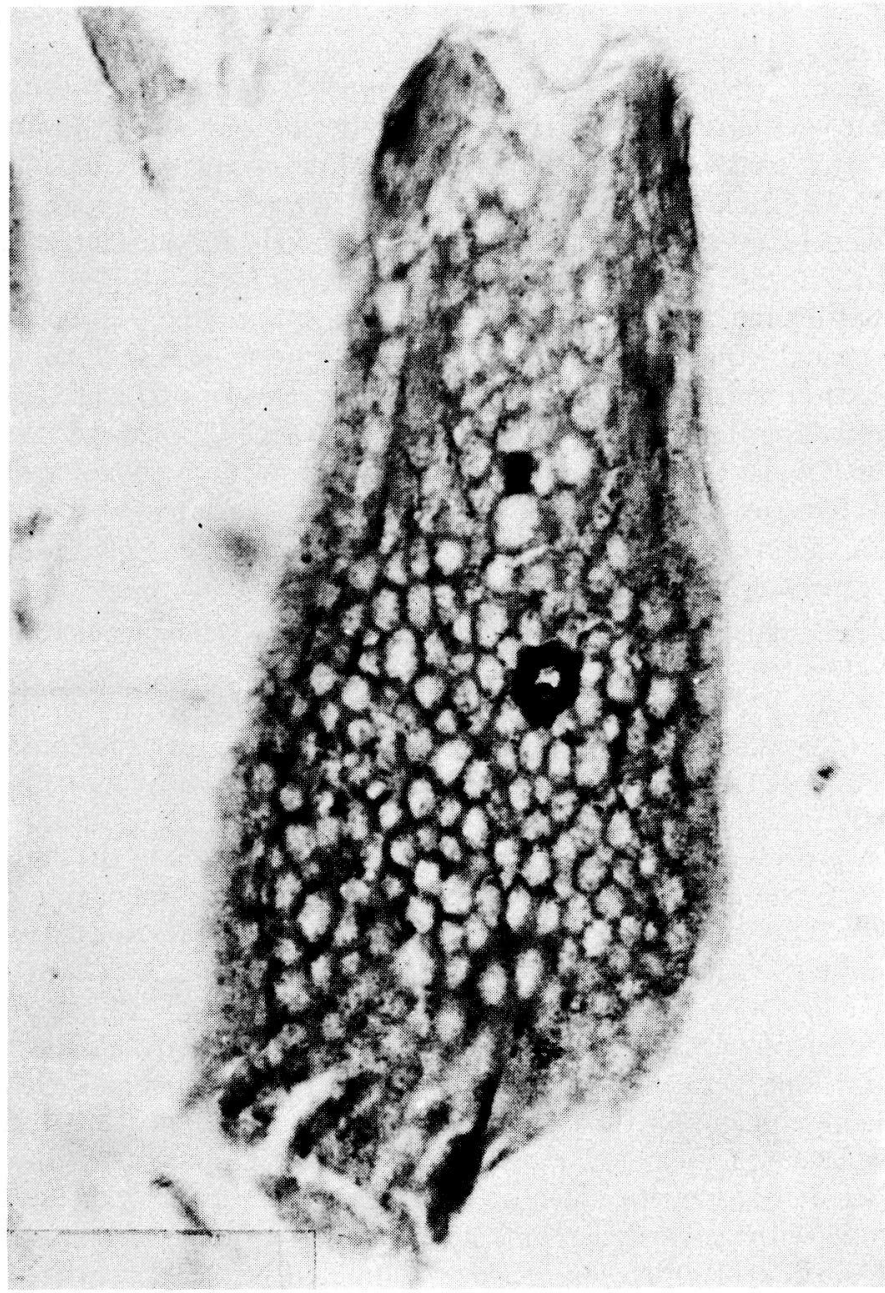

Abb. 5. Neurosekretorische Zelle aus dem Ganglion supraoesophageum. 
Das augenfälligste bei der Betrachtung des Nervensystems erscheint das Fehlen von Lobi optici. Zusammen mit der festgestellten Abwesenheit der Sehkomissur ist nicht nur die morfologische, sondern auch die volle physiologische Blindheit dieser Art bewiesen.

Sehr typisch erscheint ferner die erhebliche Konzentration des Nervensystems, das nicht aus den Grenzen des Thorax mehr herauskommt. Die Formel 2+2 / 0 scheint für Höhlenbathysciinae typisch zu sein, da es ebenfalls bei Tranteeviella bulgarica (Kwartirnikov, 1972), Netolitzkya jeanneli matroffi (Kwartirnikov, 1971), Tismanella (Decu, 1961) auftritt, sowie generell für alle Gattungen der von Decu (1960) untersuchten Unterfamilie festgestellt wird.

Die Untersuchung zeigt, daß dieser echte Höhlenbewohner normal entwickelte Pilzkörper besitzt. Dies wird um so bemerkenswerter, bedenkt man welches an Empfindungen arme Lebensmilieu diese Tiere besiedeln: Keine Lichtempfindungen, kein nennenswerter Temperatur- und Feuchtigkeitswechsel, niedrige Bevölkerungsdichte, keine Feinde. Unter diesen Bedingungen sind solche Assoziationszentren, für welche die Pilzkörper von den meisten Forschern gehalten werden, eigentlich zwecklos. Dazu kommt auch die lange Zeit, welche seit der Einwanderung in die Höhlen vergangen ist. In diesem Zusammenhang ist die beschriebene Verbindung mit dem Wurzelgebiet der Antennennerven bedeutsam. Denn obgleich die Kompensation des Lichtsinnes bei Troglobionten durch den Tast- bzw. Geruchssinn angezweifelt wird (Vandel, 1964), sprechen viele Anzeichen für eine solche Annahme. In diesem Sinne könnte auch die Verbindung der Pilzkörper mit den Wurzelgebieten der Antennennerven und letzten Endes mit den olfactorischen Loben gedeutet werden.

Über den prozentuellen Anteil der Corpora pedunculata und der olfactorischen Loben zum Gesammtvolumen des Oberschlundganglions können jedoch keine Aussagen gemacht werden, da solche Messungen in der Literatur nicht gefunden werden konnten und außerdem erst ein Vergleich mit außenlebenden Käferarten sinnvoll ist.

Die Untersuchung des endokrinen Systems hat gezeigt, daß sich im Pars intercerebralis eine beträchtliche Anzahl von neurosekretorischen Zellen befindet. Sie bleibt keinesfalls hinter dieser bei außsenlebenden Insekten (Gersch, 1964). Die neurosekretorischen Zellen des Unterschlundganglions sind beträchtlich weniger. Im Gegensatz zu den aussenlebenden Insekten treten aber in der Bauchganglienkette keine neurosekretorischen Zellen auf. Somit ist die Wirkung des Lebens unter der Erde nicht so offensichtlich wie bei dem zentralen Nervensystem. Es kann aber auch nicht zu einer weitgehenden Reduktion des endokrinen Systems kommen, da dieses die lebenswichtigsten Prozesse im Insektenleben kontrolliert.

\section{Z U S A M M E N F A S S U N G}

In der Untersuchung wird festgestellt, daß das zentrale Nervensystem die Formel 
$2+2 / 0$ besitzt. Der prozentuelle Anteil der Corpora pedunculata beträgt 5,5\% und der olfactorischen Loben 6,8\% von der Gesammtmasse des Oberschlundganglions. Das Unterschlundganglion beträgt 29,5\% von der Gesammtmasse der Kopfganglien.

Es wird weiterhin festgestellt, daß sich sowohl im Oberschlundganglion, als auch im Unterschlundganglion neurosekretorische Zellen befinden. Corpora allata und Corpora cardiaca sind ebenfalls vorhanden.

\section{R É S U M É}

Continuant sur Hexaurus merkli ses recherches sur les systèmes nerveux et endocrines des Coléoptères troglobies bulgares, l'auteur établit ici que le système nerveux central répond à la formule $2+2 / 0$. La proportion relative des corpora pedunculata atteint $5,5 \%$ et celle des lobes olfactifs $6,8 \%$ de la masse totale du ganglion sus-oesophagien. Quant au ganglion sous-oesophagien, il représente $29,5 \%$ de la masse totale des ganglions céphaliques.

Il est en outre établi que, aussi bien dans le ganglion sus-oesophagien que dans le ganglion sous-oesophagien, il existe des cellules neurosécrétrices. Des corpora allata et des corpora cardiaca sont également présents.

\section{I T E R A T U R}

DECU, V. 1961. Contributii la studiul morfologiei interne la coleopterele cavernicole din seria filetica Sophrochaeta Reitter (Catopidae, Bathysciinae). Studii si cercetari de Biologie, Seria Biologie Animala, T. XIII, Nr. 3. 395-408.

DECU, V., PAPACOSTEA, P. Anatomische und mikrobiologische Untersuchungen über einige Arten von höhlenbewohnenden Coleopteren aus Oltenien (Rumänien). Rowartani közlemenyek, Folia entom. hung., T. XVII, Nr. 6. P. 87-112.

GERSCH, M. 1964. Vergleichende Endokrinologie der wirbellosen Tiere. Leipzig, Akademische Verlagsgesellschaft Geest. Portig. K.-G.

KWARTIRNIKOV, M. 1971. Untersuchungen über das Nerven- und Endokrinesystem von Netolitzkya jeanneli matroffi Jeannel (Coleoptera, Bathysciinae). Bull. Inst. Zool. Acad. des Sciences de Bulgarie, T. XXX.

KWARTIRNIKOV, M. 1972. Über das Nervensystem von Tranteeviella bulgarica Pretner (Coleoptera, Bathysciinae) nebst Bemerkungen über das endokrine System. Bull. Inst. Zool. Acad. des Sciences de Bulgarie, T. XXXI.

VANDEL, A. 1964. Biospeologie. La Biologie des Animaux cavernicoles. Gauthier - Villars Editeur. Paris.

WEBER, H. 1954. Grundriss der Insektenkunde. Gustav Fischer Verlag. Stuttgart. 